\title{
Notes on the "Self-Centered" Factor, Based on Data from Child Language Acquisition
}

\author{
Velina Slavova \\ New Bulgarian University, Sofia, Bulgaria \\ Corresponding author. E-mail: vslavova@nbu.bg
}

Background. This study is based on large samples of language-data collected during the formative period of language acquisition (9-62 months) from free dialogues of English and French children. Analysis of the longitudinal development of children's vocabularies expressed in terms of Parts of Speech showed that their acquisition develops surprisingly differently in these two languages. The hypothesis of the study is that first-language acquisition should respect identical principles, independently of the language acquired.

Objective. To analyze the observed properties of language acquisition data based on a general model of concept formation and information processing.

Design. Two models were applied: (a) Model of information processing based on reasoning concerning information, entropy, energy optimization, and evolution; and (b) Model of native semantic roles, based on the psychological approach of grounded cognition. The common factor in the reasoning applied in these models is that the process of concept formation is based on information generated by the biological system itself.

Results. A joint formal representation is obtained using these two models. The derived mathematical formalism showed remarkable agreement with the sample data. It explains the gradual acquisition of the two languages as one and the same process. The formal description suggests that the acquisition of verbs is accomplished with reference to the concept of Self as Actor, acting in the Environment.

Conclusion. The performed analysis is in support of the hypothesis that there exist inborn mechanisms of concept formation. The investigation of the joint model suggests that the concept of Self plays a central role in the language faculty.

\section{Keywords:}

language acquisition, corpus analysis, mental representation, concept formation, Self 


\section{Introduction}

This study is based on the author's formal analysis of data pertaining to acquisition of two languages, English and French (cf. Slavova, 2017, for full details). The statistical treatment made evident a very high mathematical similarity between the acquisition processes. The data analysis revealed that it is impossible to explain the mathematical "fit" by applying reasoning based solely on language ingredients (parts of speech, syntactic structures, etc.). This prompted a general approach to concept formation, aiming to explain the statistical result by means of models based on a larger scale of factors. The question addressed here concerns the relationship between meaning as a product of mental computations, and language. The reasoning applied relies on the widely accepted psychological model proposed by Barsalou (2003), following which the mental representation of the world replicates the frame of an Actor, acting in the Environment. This led to a generalization of the Parts of Speech (POS), expressing them as belonging to classes of meanings with different cognitive complexity. These meaning-classes can be seen as semantic prototypes of syntactic categories. From this point of view, the study's overall approach can be seen as a statistical investigation related to the "semantic bootstrapping hypothesis" proposed by Pinker (1987).

The notion of the existence of inborn semantics is not new. For example, Wierzbicka (1996) proposed that there exist "semantic primes" which are universal for all languages. Such approaches investigate concrete concepts, proposed to be primary for mentally representing the world. However, the mental capacity to "calculate" the meaning of the concepts has not been investigated in previously proposed models. The principles related to information processing following which the mental representations underlying the language faculty arise and are implemented in language have still not found an explanation. The present work is an attempt to make a step towards clarification of these questions.

\section{Methods}

\section{Goal and Tasks of the Research}

The goal of the study is to investigate the mechanisms of concept formation that assist children at the initial moment of language acquisition. The study aims at discovering information models of concept formation that can explain the statistical observations of data pertaining to acquisition of two languages. Next in this paper, an analysis is proposed concerning the role of self-referential information with regard to its importance for concept formation.

\section{Research Design and Procedure}

The general steps performed to find similarities in the language-acquisition process of the two sampled language-groups of children, briefly reported here, are:

1. Annotated data for the initial period of acquisition is organized into a form treatable for further comparisons.

2. A model is built, based on the psychological model proposed by Barsalou (2003), which discriminates what "type of thing" children were thinking when saying the words. 
3. A hierarchical model of conceptualization mechanisms is built. It represents the process of creating information units, which ensures maximal entropy (i.e., delivered information-content) with minimal operational load.

The two models suppose that information is created with an active and constant inclusion of self-referential information. This self-referential mechanism is further explained and analyzed.

\section{Description of the Samples}

Data from 42 corpora containing 1,515 free dialogues with children's speech in English and French were extracted from CHILDES (Child Language Data Exchange System, see, e.g., MacWhinney, 2014) and used for statistical analyses (Slavova, 2016). The dialogues in the source were collected, transcribed, and annotated with POS by researchers in language acquisition. The results for two large corpora collections - 125,873 child sentence-utterances for English and 153,824 for French were treated statistically. The E dialogues (with 62 girls, 66 boys, and 7 children with gender not specified in the nglish data collection contains 620 source); the French collection contains 895 dialogues (with 157 girls and 141 boys). It is important to underline that in the examined samples, children express themselves in free dialogues where they wish to communicate their thoughts, desires, and intents. The majority of the stored utterances are grammatically incorrect sentences, where children structure their expressions as well as they can in order to convey their ideas and wishes. The children are between 9 and 62 months old. They use, with mistakes, the learned language-labels to express the meaning that exists in their mental world. Despite such mistakes, an important assumption in this study is that the collected data are thoroughly relevant for studying the process of concept formation.

All the pronounced utterances in the obtained English and French data collections were treated as follows:

1. For studying the use of POS, the statistical analysis relies on the annotation performed by the authors of the respective corpora.

2. To reflect one main assumption of the model, the utterances were annotated for the present study with an additional feature: "self-centered" speech. The following two cases were considered: (a) the utterance contains the pronouns "I", "me, "mine", "my", or "myself"; (b) the utterance is not wellpronounced; the pronoun is missing, but should be one of those above.

In the first months of speech, children often use verbs without the pronoun "I", as if it were clear that the only possible actor is the speaking child (children also use their own name or, for example, "baby" to refer to themselves). After 36 months, such hidden use of self-centered speech is rarely encountered in the examined data collections. These "hidden Self" utterances were retrieved and manually annotated.

The quantitative analysis of the use of POS was obtained using a measure for the contribution-weight of a given POS within a speech-utterance. The following formula for calculating the Ratio per Utterance (RU) of the given POS within a dialogue was applied:

$$
R U\left(P_{i j}\right)=\frac{N P o s_{i j}}{N_{j}}
$$


where: $P O S_{i}$ is one of the POS annotated in the corpora, $j$ is the dialogue, $N_{j}$ is the number of utterances with recognizable POS in the dialogue $j, N P o s_{i j}$ is the number of the $P O S_{i}$ in the dialogue $j$. The Ratios per Utterance show the extent of use of the given POS for expressing the child's thoughts within a communication utterance "averaged" for the dialogue.

\section{Analysis of the Data Samples}

The statistical analysis showed that the developmental paths for the use of identical POS in the speech produced by English-acquiring and French-acquiring children are astonishingly different (Slavova, 2017). The average between-languages correlation in the advancing use of identical POS is relatively small - only 0.46 (since POS frequencies on age functions are monotonically rising, the between-language correlations are typically inflated). The question was how to capture the mental representations expressed in the children's words, so as to allow identification of shared principles in constructing a representation of the world.

a. Nouns, Ratio per Utterance
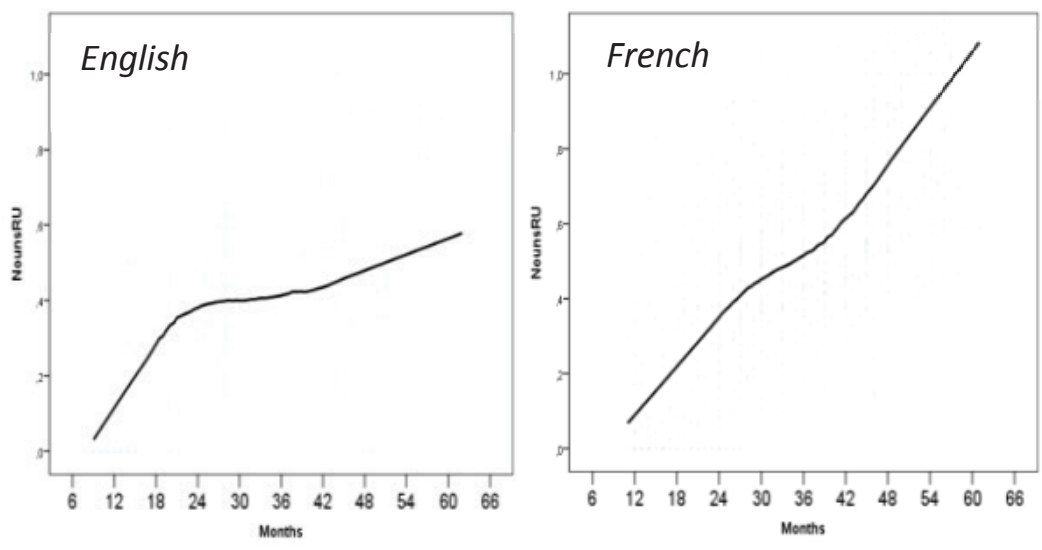

b. Verbs, Ratio per Utterance
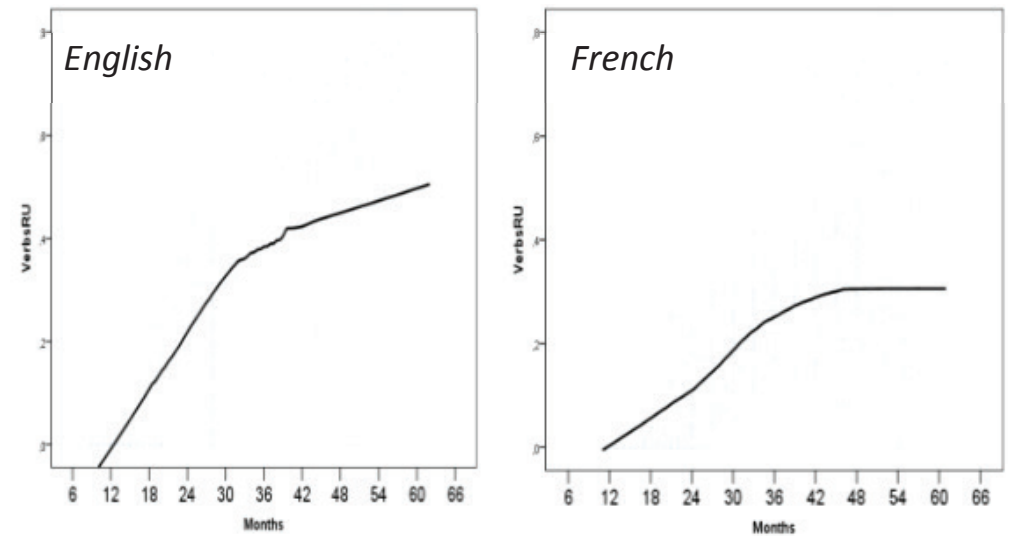

Figure 1. Use of nouns and verbs, age 9 to 62 months

The most basic grammatical categories - nouns and action verbs - have, at least within the Indo-European language family, the same deep semantics. However, as shown in Figure 1, even these basic categories have very different paths of use 
for expressing the developing ideas of the English- and French-acquiring children. There is no reason to suspect that 1-, 2- and 3-year-old English and French children have very dissimilar ideas to communicate, so the hypothesis is that these ideas are spelled out by means of groups of POS. This prompted the constitution of "classes of meaning" - groups of POS with a similar role from the point of view of the child as an Actor in the Environment.

\section{Application of Mathematical Models}

Models of language can be constructed on the basis of particular sets of scientific facts. One main question related to the language faculty is that of meaning - a phenomenon for which there are several definitions and approaches. The approach proposed in my previous works (e.g., Slavova, 2018) and followed here assumes that meaning is represented in terms of "information units", which correspond to specific activations of the brain in response to the perceived world. How the brain produces meaning and how it functions in general are questions that still lack complete answers; however, the models proposed here follow reasoning based on results obtained in the brain sciences. Such results suggest that the brain is a closed system with intrinsic activity. It is known that there exist neurons with intrinsic oscillatory capabilities and that "the brain self-generates dynamic oscillatory states which shape the functional events elicited by external sensory stimuli" (Llinás \& Paré, 1991). Based on a large set of facts from the domain of the brain sciences, Llinás (2008) concludes that: "subjectivity is what the nervous system is really all about, even from the most primitive levels of evolution”. Llinás (2001) furthermore posits that all perceptual and cognitive faculties evolved expressly so as to optimize animals' ability to generate actions required for surviving in their environment.

The modeling approach adopted here reflects this self-generating property of the brain, seen as a closed system with intrinsic activity, as well as the phenomenon of subjectivity, which appears deeply encoded in the functioning of the nervous system.

\section{The Actor in the Environment Model}

This is a model of categories of information or "classes of meaning" that humans create about the world. The classes of meaning proposed (Slavova, 2017) are the products of general reasoning as to the character of the information that has to be managed by a hypothetical autonomous system displaying complex, goal-oriented behavior as Actor in the Environment. This led to the following classes: Entities, Relationships, Circumstances, Quality and Attribution, Quantity and Precision, and Other. A separate Entity is attributed to Self - the Actor.

In order to statistically test the model's reliability, children's expressions were categorized according to the proposed classes of meaning. The statistical data were used as sources to obtain metadata corresponding to the model. The application of the model made evident that the two language-acquisition processes are very similar (Figure 2). The pattern of acquisition of the meaning-classes displayed a high between-languages class-to-class correlation (Pearson's $r=0.78, p<0.001$ ). Examination of the data showed that all the meaning-classes start being used from the outset of speech production, that is, at 10-14 months. This indicates that the semantics of the proposed classes is fundamental (Slavova, 2017). 

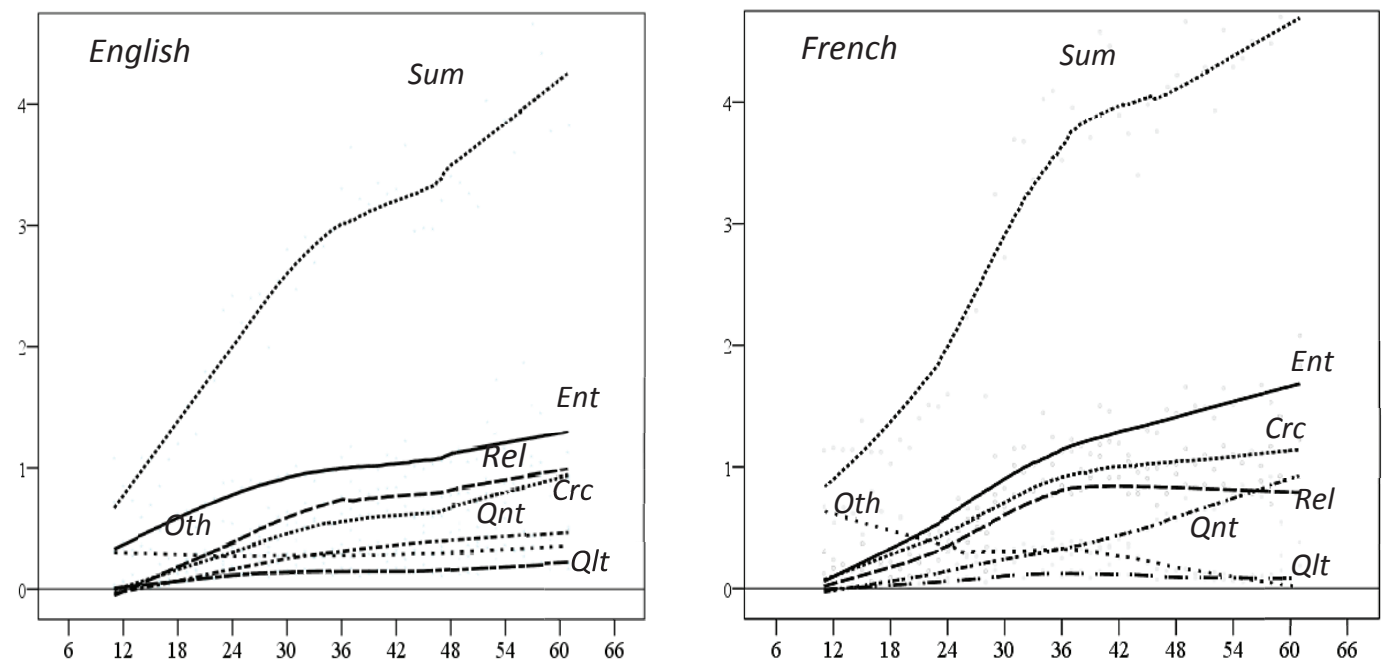

Figure 2. Use of the meaning-classes, age 9 to 62 months (Slavova, 2017).

Note: Ent $=$ Entities, Rel $=$ Relationships, Crc $=$ Circumstances, $Q$ nt $=$ Quantity, $Q l t=$ Quality

The statistical analysis showed that the rates of acquisition of the different meaning-classes are different (Figure 2). For example, Entities are acquired much more intensively in both languages - so, are Entities more easily conceptualized? This suggests that the information processing involved in the conceptualization of the different classes of meaning has different levels of complexity. The further investigation has necessitated the construction of a mathematical model of information processing. That proposed and used here (Slavova, 2018) is based on wellknown principles in physics and information theory. The mathematical approach applied aimed at finding an optimal solution when maximizing the entropy (the information) and minimizing the energy cost of the information treatment.

\section{The Maximizing of Entropy Model}

One basic assumption in the Maximizing of Entropy information-processing model (previously proposed, see Slavova, 2018) is that information is created by means of a joint treatment of two signals - one coming from the environment, and the other generated within the organism. This led to an information-treatment model in the form of a binary processing tree. The creation of information was presented as a result of a binary operation of "information gathering", called i-Merge. Each i-Merge operates on a node of the tree by detecting disparity. Operation i-Merge has two inputs - one exogenous and one endogenous. Both convey encoded information units (this is called an information flow). Each i-Merge produces a novel information unit (novel information flow) based on the dissimilarity between the two incoming information flows.

The bottom-up successive i-Merges form paths that underlie a process of generating more complex levels of information. It has been shown (Slavova, 2018) that such processing obtains maximum classified information with minimal processing cost (energy) when it respects the settings of a theorem demonstrated by Horibe $(1982,1983)$. Horibe showed that in a binary tree with $n$ terminal nodes weighted with probabilities $p_{1}=p_{2}=\ldots=p_{n}, \sum p_{i}=1$, the Shannon entropy is maximized, with minimal average cost, by a Fibonacci tree. 
The obtained processing tree is presented in Figure 3. The information processing over the tree is bottom-up. There are two categorical sources of information exogenous, coming from the environment, and endogenous, providing information intrinsic to the biological system itself, including memory. The terminal nodes represent inputs from multimodal perception (coupled exogenous-endogenous) and memory. The entire information processing relates these sources in a joint information channel.

This formal mathematical expression of the principle "maximal entropy with minimal energy" became possible due to the inclusion of the information flow created by the system. This information flow is created by the processes pertaining to the system's own functioning. As a result, the model describes the gradual assembling of information units over the processing hierarchy as being "Self-referential". This led to naming the obtained formalism a "Self-centered" model.

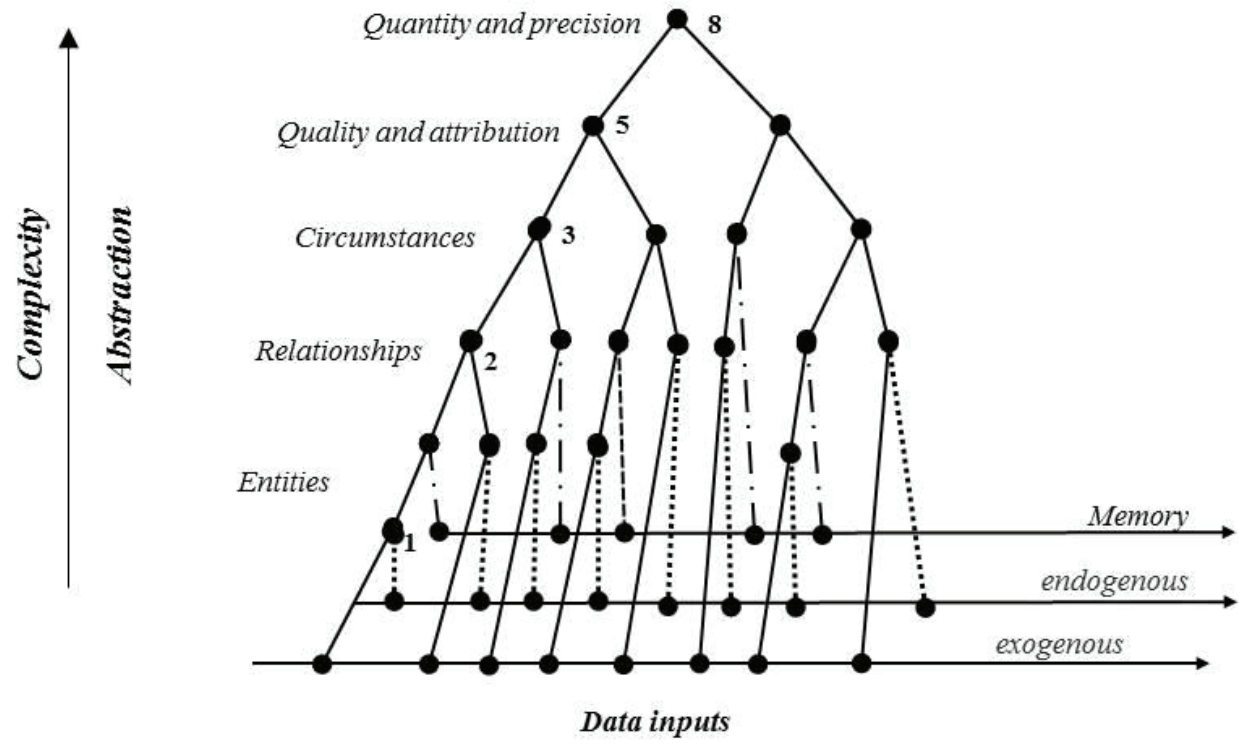

Figure 3. Information treatment model (Slavova, 2018).

\section{Results}

\section{Convergence of the Applied Models and the Child-Speech Data}

To relate the information-processing model to the dissimilar complexity of the meaning-classes (Figure 2) that became evident after the statistical analysis of the child-speech data, the hierarchical information processing (Figure 3) was expressed in terms of computational complexity. A measure of the processing load was determined as follows: The cost of each level has a value equal to the number of primary information units (i.e., perceived sensory information) entering the corresponding sub-tree through the exogenous input. The meaning-classes inferred from the Actor in the Environment model were placed in the successive levels of the hierarchical processing. As shown in Figure 3, the cognitive processing loads attributed this way are: Entities $=1$, Relationships $=2$, Circumstances $=3$, Quality $=5$, and Quantity $=8$. This order (Slavova, 2018) signifies the level of abstraction of each meaning-class. Entities are directly based on multimodal perception. Relationships 
are concepts about the actions and states of Entities, and obtain a concrete meaning when referred to Entities. Circumstances include spatial and temporal characteristics of Entities and/or Relationships. Quality and attribution require extracting of similar features from Entities, Relationships, and Circumstances, and blending them in separate concepts. Quantity and precision need the establishment of concepts that estimate proportions, grouping of Entities, or selecting one Entity from a conceptualized group.

The derived complexity of the classes was used for verification of the model with the statistical observations. The complexity of the concepts (CC) used in the children's speech by the two language groups was calculated as follows:

$$
\mathrm{CC}=1 \cdot E n t+2 \cdot \mathrm{Rel}+3 \cdot \mathrm{Crc}+5 \cdot \mathrm{Qlt}+8 \cdot \mathrm{Qnt}
$$

where: Ent, Rel, Crc, Qlt, and Qnt are the statistical results for the Average Ratio per Utterance of the use of classes of meaning $\left(A R U M_{e, f}\right)$ proposed in the Actor in the Environment model, where $\left(A R U M_{e, f}\right)$, for the two languages correspondingly English and French - is calculated with the equation:

$$
\operatorname{ARUM}_{e, f}\left(C l_{i}\right)=\frac{\sum_{m=1}^{M_{e f}}\left(\frac{N C l_{i, j}}{N_{j}}\right)}{M_{e, f}}
$$

where $C l_{i}$ is one of the classes of meaning (Ent, Rel, Crc, Qlt, and Qnt) used by the children, $j$ is the dialogue, $N_{j}$ is the number of utterances in the dialogue $j, N C l_{i j}$ is the number of the words from the class $\mathrm{Cl}_{i}$ in the dialogue $j, M_{e, f}$ is the number of dialogues, and the indices $e$ and $f$ are for the English and the French data collections, respectively. The Ratio per Utterance of a class of meaning $N C l_{i j} / N_{j}$ shows the extent of use of the given class for expressing the children's thoughts within a communication utterance "averaged" for the dialogue.

The application of this model-derived equation to the statistical observations led to a very high between-languages correlation $(r=0.933 ; p<0.01)$ for the development of language production. The hypothesis (in statistical terms) that such a between-languages correlation could be obtained by chance was rejected at $p<0.01$ via Monte-Carlo bootstrapping (Slavova, 2018).

This model offers a hint as to the principle whereby information arises in living matter. The analysis and reasoning provided in Slavova (2018) have proposed that the internal creation of information uses internal "prototypical" signals to create information units about the Actor's environment. This suggestion is additionally supported by a very recent theory in neuroscience, the "Neural Self-Information Theory", postulating that neurons generate synchronized spiking as "Self-Information" codes (Li et al., 2018). The described approaches used for the models are different, but converge, as shown in Figure 3, to a joint representation that explains data from language acquisition of the two languages as one and the same process.

\section{Model of the Process of Conceptualizing Verbs}

As seen in Figure 3, the model suggests that concepts can be formed only with active inclusion of an internally generated self-referential information flow. A fundamental question in this representation is what is "given" as a self-referential information flow at birth, such that the brain can start constructing a semantic rep- 
resentation of the world. The main model-based hypothesis which follows is that the verbs are concepts that are strongly related to the concepts of Self. The reasons for this hypothesis are briefly explained below. The mental representations that correspond to the recent findings discussed below are referred to as the concepts of "Self" and "Self-similar".

\section{Analysis of Findings from Brain Sciences}

A previous analysis (Slavova \& Soschen, 2015a) led to the conclusion that genetically determined information processing realizes the concept of Self by means of proprioception, interoception, processing by the Mirror Neuron System (MNS) (e.g., Rizzolatti \& Craighero, 2004) and by the Default Mode Network (DMN) (e.g., Horn, Ostwald, Reisert, \& Blankenburg, 2013). Numerous brain studies have been conducted toward identifying the processes by which the concept of Self is established. For example, Davey and colleagues (2016) suggest that a composite "brain-Self" system is responsible for engendering conscious self-awareness which provides the sense of oneself as a subjective Agent. The effort to understand brain functioning related to the concept of Self has led to "Countless functional neuroimaging studies..." (Tsakiris, 2017), which have reported various brain structures and processes related to the concept of Self. The result of these efforts has not still provided a definitive explanation for the phenomenon "Self", but they all indicate the existence of brain mechanisms underlying a precise mental representation associated with what we have termed the concept of Self.

Other brain-imaging results (e.g., Connolly \& Haxby, 2012; Molnar-Szakacs \& Uddin, 2013a,b) show that the brain reacts in a specific way to perceptual features that are "Self-like" (including of animals, especially mammals). Recent fields in brain research such as the "social brain sciences" (Adolphs, 2003) have revealed neurobiological links between the Self, emotion, and society. Other results report an overlap between the DMN and brain areas mediating social cognition (e.g., Mars et al., 2012). Such efforts led to another discovery: that the brain mechanisms associated with the concept of Self are related to a specific conceptualization ability - the understanding of the Self as a part of a society or, in other words, the availability of a categorical concept corresponding to the Self-similar. In a sense, these research areas may be reduced to the question, "How do humans recognize conspecifics?" Papeo and colleagues (2017) interpreted this question as asking "what it means to recognize humans" and found, based on brain imaging, that this ability involves brain patterns treating perceptual characteristics of conspecifics and the retrieval of more abstract information that determines category membership (human or nonhuman). The brain mechanisms that correspond to such recent findings suggest the existence of a biological basis underlying the concept of "Self-similar".

\section{Modeling the Conceptualization of Verbs}

The model's processing tree (Figure 3) suggests that the creation of an information unit corresponding to an Entity can be accomplished solely by the neural substrates of multimodal perception. This is not the case for the Relationships (Verbs). Verbs, as the model suggests, necessitate additional memory input. For 
instance, analysis of adults' fMRI results led Di Cesare and colleagues (2017) to conclude that an action verb "prepares us to interact with an object even though no object name is presented in our linguistic stimuli (the object is very likely inferred by the participants)". This suggests that conceptualization of action verbs uses semantic memory.

The assembly of larger volumes of mentally operable information depends on the capacity to manage the argument structure of a sentence. It was shown on the basis of data from the English collection (Slavova \& Soschen, 2015b) that the assigning of thematic roles in children's sentences occurs gradually with age, by means of the successive inclusion of sentence arguments as follows: 1 . S-V; 2. V-O; 3. S-V-O; 4. S-V-O-O. The important observation was that the first uses of these argument frames start, for each of the enumerated structures, by composing sentences in which the Self is the Subject. The observed intensive use of expressions with the Self as Actor suggests that verbs are assisted by "mirroring" of perceived actions of the Self-similar.

The term "mirroring", following its use in the specialized studies of MNS (e.g., Mihov et al., 2013), denotes the automatic process initiated by the MNS, consisting of various types of activation of the person's own body and emotional state in correspondence with that observed in other humans. Concerning the acquisition of verbs, the hypothesis suggested by the information-processing model is that when observing other people's actions, mirroring creates a mental activation which corresponds to one's own action, accorded to the concept of Self. This assists the creation of a concept "ready to use" in speech, where the Self is the Actor in the sentence (in accordance with the Actor in the Environment model). This supposes that the mirroring assists children in the creation of concepts for verbs and that the process is based on the concepts of Self and the "mirrored" Self-similar.

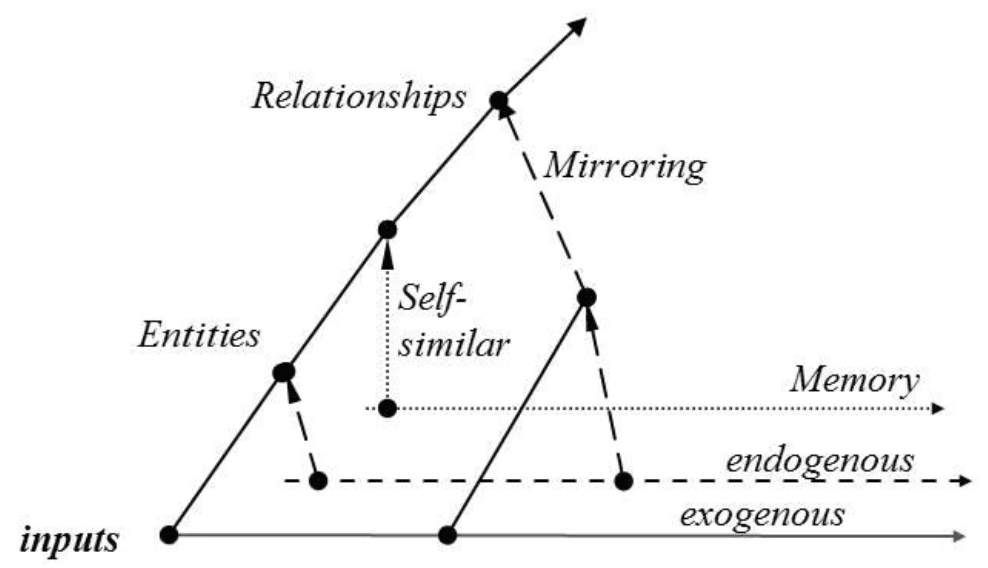

Figure 4. Mirroring of the Self-similar to conceptualize verbs.

In compliance with all the enumerated data, the Maximizing of Entropy model is further developed in the context of mirroring (Figure 4). Relationships (verbs) are conceptualized on the basis of three types of inputs: the coupled exogenousendogenous inputs, creating a perceptual unit, and an input conveying memorized Entities. This scheme proposes that the inputs facilitating the conceptualization of verbs necessitate the perception of actions of the Self-similar in order to match 
them to the Actor's own actions and intents. At the time of birth, an infant's semantic memory is (at least) very meagre, as the newborn has still not experienced the outside world. Following the scheme in Figure 4, the information processing required for conceptualizing relationships cannot ensue without a representation of the Self-similar. The model suggests that the awareness of the Self-similar is genetically underpinned.

This model's prediction is supported by several studies of newborns' reactions to human faces or voices, suggesting that such awareness exists at birth. As an example, Streri and colleagues (2013) demonstrated that just hours after birth, human infants are capable of imitating human facial movements. These authors propose that there exists an innate predisposition to social interactions in newborns. Although it is difficult to investigate the neurobiological basis of such phenomena, novel and adapted fMRI methods make it possible to suggest an answer to the question: Does the concept of Self-similar reflect innate predispositions? A recent fMRI study by Deen and colleagues (2017) demonstrated that, while the anatomical maturation of the human cortex is slow and asynchronous, by 4-6 months, human infants' visual cortex already has a spatial organization mimicking that of adults, mediating category-sensitive responses to human faces and to environmental scenes.

\section{Discussion}

\section{Note on Piaget's "Egocentric Stage"}

The Self-centered characteristic of the model presented here is not related to the "egocentric stage" described by Piaget (e.g., Piaget, 2015). Piaget notes that the incidence of egocentric speech slowly dies out between 2 and 7 years as that of "social speech" increases, where the intent of social speech is to communicate with the social environment.

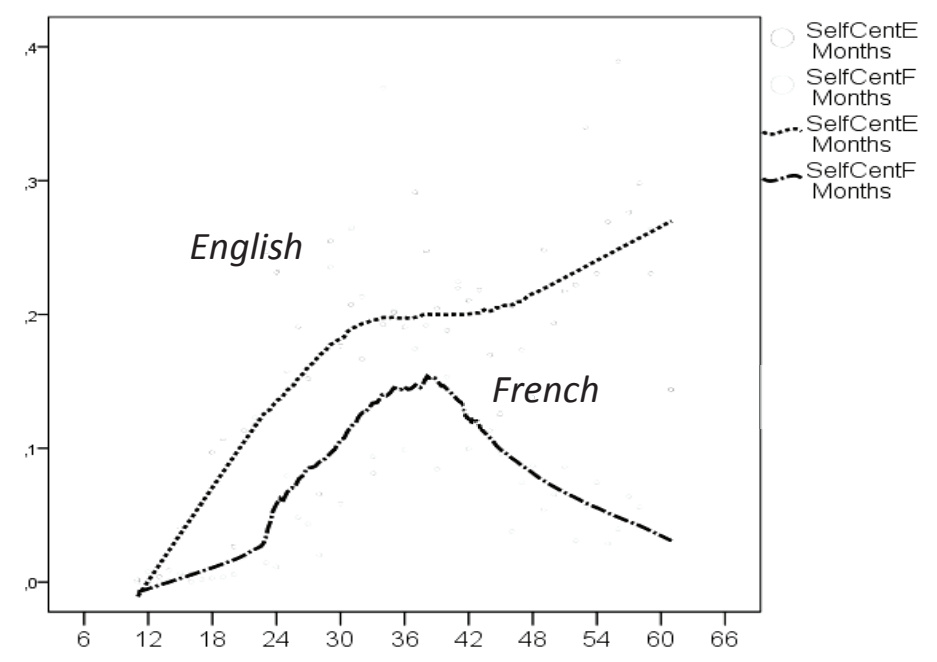

Figure 5. Self-referencing, as measured in speech

According to the present study's reasoning, children try to make their communication as successful as possible from the very outset. The fact that children start expressing, for example, Entities with the words used in the language environment 
represents already a sign of their communicative intent. As mentioned earlier, in order to follow the progress in the structure of sentence arguments, an additional data annotation was performed to evaluate the child's use of explicit or hidden references to himself or herself.

This provides one more Ratio per Utterance, the Self-Referencing Ratio, showing the participation of pronouns and names that designate the speaking child. The development of this Ratio is shown in Figure 5. The two language groups show a different developmental path in the use of self-referenced speech. After 36 months, the French group utters fewer and fewer self-referenced expressions. The English group does not show a decrease of this use, but rather a period of lack of increase.

If one assumes that the use of self-referencing is a sign of egocentric behavior and that the particularities of the two languages do not influence the use of selfreferencing (which does not seem true), the English group does not correspond to the observation of Piaget. However, as seen from the plot presented in Figure 5, for the period 11-36 months, the two language groups show a net tendency to advance in speech production when referring to themselves (for this period, the betweenlanguages Pearson's $r$ is $0.586, p<0.001$. The self-referencing stage, from the point of view of the model here, is related to the initial frame for representing the world, in which the Self is the Actor. The hypothesis that can be considered for the later period is that after 36 months, children start being strongly influenced by everyday vernacular usages of language typical among older speakers.

\section{Some Comparisons with Findings from Child Language-Acquisition Studies}

Studies of children's language acquisition do not concentrate explicitly on the parameters that are involved in the model presented here; however, examination of the reported studies in this field can identify several findings that are supportive of the model.

The main hypothesis concerning verb acquisition implied by the model pertains to children's awareness of the Self and the Self-similar. More precisely, the hypothesis is: When the child can perceive an action performed by a Self-similar, perception and mirroring "automatically" map it onto the child's inborn information flows, assisting the creation of the verb concept. To produce sentences with the Self as actor necessitates one single mental step after the automatic mirroring: the use of the verb with its conventional public label. Such a scheme leads to the supposition that actions that are not observed as performed by the Self-similar should be more difficult to conceptualize. When the child has to give a name to a self-formulated action that has never been observed as performed by a Self-similar, the conceptualization will lack the input necessary to directly prompt the mirroring. In this case, two mental steps will be needed for creating the verb concept: step $1-$ (mental) projecting of one's own action onto a Self-similar Entity; step 2 - mirroring the imagined action of the Self-similar in order to prompt the automatic creation of the verb concept.

Studies in verb acquisition are supportive of this hypothesis. Childers and Tomasello (2006) examined children's comprehension and production of new verbs using several conditions, including training with either the Self-agent or a Self-similar puppet agent. The result showed that "when children initially heard a new verb that referred to their own actions, they were better at responding to questions that referred to their own actions than questions that referred to another agent's actions. 
This was not true of the verbs in which children saw a puppet agent". (Childers \& Tomasello, 2006). Other studies of language acquisition resulted in further findings related to the Self-similar hypothesis. For example, Mandler (2006) showed that 14-month-old infants typically refuse to imitate actions that are contextually inappropriate, such as putting a toy vehicle to bed or giving it a drink.

Following the present model, it can be supposed that verbs that denote actions of a Self-similar are more easily conceptualized and will outnumber the other verbs in children's speech. Indeed, the results of Buresh and colleagues (2006) showed that verbs describing events that involve actions by other people are prevalent in children's vocabularies. These authors suggest that "understanding the actions of others is critical for discerning their communicative intentions, and thus provides a foundation for all aspects of language learning, not just verb learning".

\section{Evolution and the Concept of Self}

A further question is whether the information processing that assists the mental representation of the world from the standpoint of a goal-oriented Actor who has an idea about the Self is uniquely human. It is not currently clear how exactly the awareness of Self emerged in the course of evolution, but it is known that some non-human primates (namely the great apes) successfully pass the standard "mirror self-recognition test" (e.g., Anderson \& Gallup, 2015; Gallup, 1970; Robert, 1986; Westergaard \& Hyatt, 1994) as well as some non-mammalian species (e.g., Delfour \& Marten, 2001). This is strong evidence of self-recognition in animals and indicates the possibility of self-awareness ("sense of self"), but not a definitive proof. Concerning the neurobiological aspects of the phenomena related to the awareness of the Self and of the Self-similar, it should be noted that the MNS, originally discovered in macaques, has been recognized in several other species (see, e.g., Rizzolatti \& Craighero, 2004). As regards the DMN, crucial for mediating introspection, a number of studies (e.g., Vincent et al., 2007) discovered that it exists in nonhuman primates. Mars and colleagues (2012) propose that many facts

revealed by studies of DMN in nonhuman primates show an evolutionary aspect of DMN's functional organization, which in humans displays a degree of overlap with areas that mediate social cognition.

There are reasons to believe that the mental mechanisms discussed here arose in pre-human evolutionary development and exist to some extent in nonhuman primates. Accordingly, there are no reasons for nonhuman primates not to develop such an elaborated language as that of humans. But they did not. To find the precise reasons necessitates serious efforts in several domains.

So, for the moment it is not clear whether only humans have a concept of Self. However, an anthropological model of evolution recently proposed by Messori (2016), exploring evidence and results from several anthropological investigations, advises that the conceptualization of the Self as a Subject within a subject-environment relationship (Subject-Object, as presented there) triggered the language faculty.

\section{Conclusion}

The examined speech data and the hypothesis prompting the various forms of analyses undertaken by me here and elsewhere, led to the inclusion of a Self-referential parameter in the proposed model. The application of this resulting Self-centered 
model showed remarkable convergence with the data and explains child language acquisition of the two languages as a common process. The model proposes that verbs are conceptualized by means of hierarchical information processing reliant upon the concepts of Self and Self-similar. The model advises that the establishment of these concepts is assisted by phylogenetically transferred information.

\section{Limitations}

This model and the derived conclusions are based on already-annotated data collected from two language samples. The annotated child dialogues used are available in the CHILDES data repository. The generalization of the conclusions for more languages and for human language in general can be pursued by way of including samples from other languages. For the moment, there are no other language samples in the CHILDES repository that are in themselves sufficient in size and contain the necessary annotation to perform a similar statistically reliable analysis. With the inclusion of more language samples, the statistical picture might possibly converge on the model proposed here or lead to other assumptions, models, and reasoning.

\section{Acknowledgements}

The author is grateful to Gary Mazzaferro for fruitful discussions, constructive advice, and suggested sources, and with the same regard to Richard Traub who in addition edited the text.

\section{References}

Adolphs, R. (2003). Cognitive neuroscience: Cognitive neuroscience of human social behaviour. Nature Reviews Neuroscience, 4(3), 165. https://doi.org/10.1038/nrn1056

Anderson, J.R., \& Gallup, G.G. (2015). Mirror self-recognition: A review and critique of attempts to promote and engineer self-recognition in primates. Primates, 56(4), 317-326. https://doi.org/10.1007/s10329-015-0488-9

Barsalou, L. (2003). Situated simulation in the human conceptual system. Language and Cognitive Processes, 18(5-6), 513-562. https://doi.org/10.1080/01690960344000026

Buresh, J.S., Woodward, A., \& Brune, C.W. (2006). The roots of verbs in prelinguistic action knowledge. In K. Hirsh-Pasek \& R.M. Golinkoff (Eds.), Action meets word: How children learn verbs (pp. 208-227). New York: Oxford University Press. https://doi.org/10.1093/acpr of:oso/9780195170009.003.0009

Childers, J. B., \& Tomasello, M. (2006). Are nouns easier to learn than verbs? Three experimental studies. In K. Hirsh-Pasek \& R. M. Golinkoff (Eds.), Action meets word: How children learn verbs (p. 311). New York: Oxford University Press. https://doi.org/10.1093/acprof:o so/9780195170009.003.0013

CHILDES - the child language component of the TalkBank system for sharing and studying conversational interactions. Retrieved from: http://childes.talkbank.org/

Connolly, A., \& Haxby, J. (2012). Brain activity shows that mammals are more animate than reptiles and bugs. Journal of Vision, 12(9), 1108-1108. https://doi.org/10.1167/12.9.1108

Davey, C.G., Pujol, J., \& Harrison, B.J. (2016). Mapping the self in the brain's default mode network. Neuroimage, 132, 390-397. https://doi.org/10.1016/j.neuroimage.2016.02.022

Deen, B., Richardson, H., Dilks, D.D., Takahashi, A., Keil, B., Wald, L.L., ... \& Saxe, R. (2017). Organization of high-level visual cortex in human infants. Nature Communications, 8, 13995. https://doi.org/10.1038/ncomms13995 
Delfour, F., \& Marten, K. (2001). Mirror image processing in three marine mammal species: Killer whales (Orcinus orca), false killer whales (Pseudorca crassidens) and California sea lions (Zalophus californianus). Behavioural Processes, 53(3), 181-190. https://doi.org/10.1016/ s0376-6357(01)00134-6

Di Cesare, G., Errante, A., Marchi, M., \& Cuccio, V. (2017). Language for action: Motor resonance during the processing of human and robotic voices. Brain and Cognition, 118, 118127. https://doi.org/10.1016/j.bandc.2017.08.001

Fleming, S. M., Weil, R. S., Nagy, Z., Dolan, R. J., \& Rees, G. (2010). Relating introspective accuracy to individual differences in brain structure. Science, 329(5998), 1541-1543. https:// doi.org/10.1126/science.1191883

Gallup, G. G. (1970). Chimpanzees: Self-recognition. Science, 167(3914), 86-87. https://doi. org/10.1126/science.167.3914.86

Horibe, Y. (1982). An entropy view of Fibonacci trees. The Fibonacci Quarterly, 20(2), 168-178. Retrieved from: https://www.fq.math.ca/Scanned/20-2/horibe.pdf

Horibe, Y. (1983). Notes on Fibonacci trees and their optimality. The Fibonacci Quarterly, 21(2), 118-128. Retrieved from: https://www.mathstat.dal.ca/FQ/Scanned/21-2/horibe.pdf

Horn, A., Ostwald, D., Reisert, M., \& Blankenburg, F. (2013). The structural-functional connectome and the default mode network of the human brain. NeuroImage. 102: 142-151 https:// doi.org/10.1016/j.neuroimage.2013.09.069

Li, M., Xie, K., Kuang, H., Liu, J., Wang, D., Fox, G.E., ... \& Tsien, J.Z. (2018). Neural coding of cell assemblies via spike-timing self-information. Cerebral Cortex, 28(7), 2563-2576. https://doi.org/10.1093/cercor/bhy081

Llinás, R. (2008). Of self and self awareness: The basic neuronal circuit in human consciousness and the generation of self. Journal of Consciousness Studies, 15(9), 64-74. Retrieved from: https://philpapers.org/rec/LLIOSA

Llinás, R.R. (2001). I of the vortex: From neurons to self (Vol. 50). Cambridge, MA: MIT Press. https://doi.org/10.7551/mitpress/3626.001.0001

Llinás, R.R., \& Paré, D. (1991). Of dreaming and wakefulness. Neuroscience, 44(3), 521-535. https://doi.org/10.1016/0306-4522(91)90075-Y

MacWhinney, B. (2014). The CHILDES project: Tools for analyzing talk, Vol. II: The database. Psychology Press. https://doi.org/10.1.1.259.8262

Mandler, M. (2006). Actions organize the infant's world. In K. Hirsh-Pasek \& R. M. Golinkoff (Eds.), Action meets word: How children learn verbs (pp. 111-133) New York: Oxford University Press. https://doi.org/10.1093/acprof:oso/9780195170009.003.0005

Mars, R.B., Neubert, F.X., Noonan, M.P., Sallet, J., Toni, I., \& Rushworth, M.F. (2012). On the relationship between the "default mode network" and the "social brain". Frontiers in Human Neuroscience, 6, 189. https://doi.org/10.3389/fnhum.2012.00189

Messori, C. (2016). From continuity to contiguity: On the genesis of consciousness, culture and oral language (Part III). Journal of Consciousness Exploration \& Research, 7(2). Retrieved from: http://jcer.com/index.php/jcj/article/view/542

Mihov, Y., Kendrick, K.M., Becker, B., Zschernack, J., Reich, H., Maier, W., ... \& Hurlemann, R. (2013). Mirroring fear in the absence of a functional amygdala. Biological Psychiatry, 73(7), e9-e11. https://doi.org/10.1016/j.biopsych.2012.10.029

Molnar-Szakacs, I., \& Uddin, L. Q. (2013 a). The emergent self: How distributed neural networks support self-representation. In D. Franks \& J.H. Turner (Eds.): Handbook of neurosociology (pp. 167-182). Dordrecht: Springer. https://doi.org/10.1007/978-94-007-4473-8_13

Molnar-Szakacs, I., \& Uddin, L.Q. (2013). Self-processing and the default mode network: Interactions with the mirror neuron system. Frontiers in Human Neuroscience, 7, 571. https://doi. org/10.3389/fnhum.2013.00571

Papeo, L., Wurm, M. F., Oosterhof, N.N., \& Caramazza, A. (2017). The neural representation of human versus nonhuman bipeds and quadrupeds. Scientific Reports, 7(1), 14040. https:// doi.org/10.1038/s41598-017-14424-7 
Piaget, J. (2015). The grasp of consciousness: Action and concept in the young child. London: Psychology Press. https://doi.org/10.4324/9781315722382

Pinker, S. (1987). The bootstrapping problem in language acquisition. In B. MacWhinney (Ed.), Mechanisms of language aquisition (pp. 399-441). Hillsdale, NJ: Lawrence Erlbaum Associates.

Rizzolatti, G., \& Craighero, L. (2004). The mirror-neuron system. Annu. Rev. Neurosci., 27, 169192. https://doi.org/10.1146/annurev.neuro.27.070203.144230

Robert, S. (1986). Ontogeny of mirror behavior in two species of great apes. American Journal of Primatology, 10(2), 109-117. Retrieved from: https://onlinelibrary.wiley.com/doi/ abs/10.1002/ajp.1350100202

Slavova, V. \& Soschen, A. (2015a). On mental representations: Language structure and meaning revised. International Journal Information Theories \& Applications. 2(4), 316-325. Retrieved from: http://www.foibg.com/ijita/vol22/ijita22-04-p02.pdf

Slavova, V. \& Soschen, A. (2015b). Syntactic operations - modelling language faculty. International Journal Information Theories \& Applications, 2(4), 326-337. Retrieved from: http:// www.foibg.com/ijita/vol22/ijita22-04-p03.pdf

Slavova, V. (2016, July). Data collection for studying language acquisition. In Proceedings of the 12th Annual International Conference on Computer Science and Education in Computer Science (CSECS 2016) (pp. 119-127). Retrieved from: https://www.ceeol.com/search/articledetail?id=529683

Slavova, V. (2017). On native semantic roles: Comparative study based on data from child language acquisition of English and French. International Journal of Cognitive Research in Science, Engineering and Education, 5(2), 1-18. https://doi.org/10.5937/ijcrsee1702001s

Slavova, V. (2018). Information-processing model of concept formation - Is first language acquisition universal? Cybernetics and Information Technologies,18(3), 3-22. https://doi. org/10.2478/cait-2018-0035

Streri, A., Coulon, M., \& Guellaï, B. (2013). The foundations of social cognition: Studies on face/voice integration in newborn infants. International Journal of Behavioral Development, 37(2), 79-83. https://doi.org/10.1177/0165025412465361

Tsakiris, M. (2017). The multisensory basis of the self: From body to identity to others. The Quarterly Journal of Experimental Psychology, 70(4), 597-609. https://doi.org/10.1080/174 70218.2016.1181768

Vincent, J.L., Patel, G.H., Fox, M.D., Snyder, A. Z., Baker, J.T., Van Essen, D.C., ... \& Raichle, M.E. (2007). Intrinsic functional architecture in the anaesthetized monkey brain. Nature, 447(7140), 83. https://doi.org/10.1038/nature05758

Westergaard, G.C., \& Hyatt, C.W. (1994). The responses of bonobos (Pan paniscus) to their mirror images: Evidence of self-recognition. Human Evolution, 9(4), 273-279. https://doi. org/10.1007/bf02435514

Wierzbicka, A. (1996). Semantics: Primes and universals. UK: Oxford University Press.

Original manuscript received October 14, 2018

Revised manuscript accepted January 10, 2019

First published online March 15, 2019

To cite this article: Slavova, V. (2019). Notes on the "Self-centered" factor, based on data from child language acquisition. Psychology in Russia: State of the Art, 12(1), 172-187. DOI: 10.11621/ pir.2019.0113 